\title{
Auch bei COPD kann man Betablocker einnehmen - aber nicht alle
}

\author{
Komorbidität kann eine leitliniengerechte Medikation verhindern. Oft erhalten Patienten mit \\ Herzinsuffizienz keinen Betablocker, weil sie auch an einer COPD leiden. Eine Studie zeigt nun, \\ dass diese Vorsicht wohl übertrieben ist. Der Betablocker sollte aber klug gewählt werden.
}

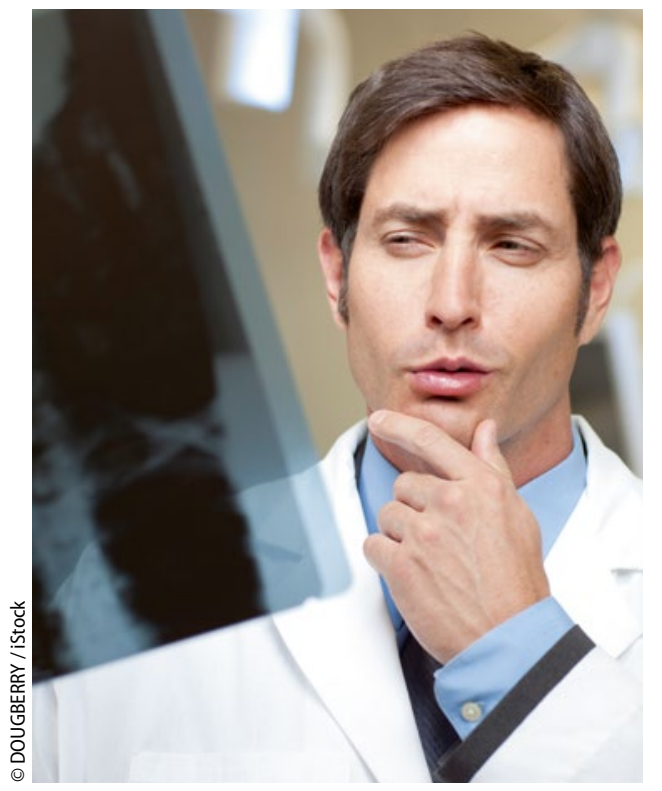

COPD oder Asthma? Das ist auch bei der Betablocker-Abwägung wesentlich.

COPD ist einer der wesentlichen Gründe für die Unterdosierung von Betablockern bei Patienten mit Herzinsuffizienz. Der Grund liegt in der Sorge, dass die Medikamente die Atemwegssituation verschlimmern könnten. Doch wie beeinflussen Betablocker tatsächlich den Verlauf einer Herzinsuffizienz bei Patienten mit COPD? Und gibt es Unterschiede zwischen den verschiedenen verfügbaren Betablockern?

Diesen Fragen ging eine monozentrische, nicht randomisierte, retrospektive Beobachtungsstudie nach. Untersucht wurde der Verlauf bei Patienten, die wegen akuter Dekompensation einer Herzinsuffizienz stationär behandelt wurden und gleichzeitig an einer COPD litten. 86 Patienten erhielten bei der Entlassung aus dem Krankenhaus Betablocker (52 Carvedilol, 34 Bisoprolol), 46 Patienten erhielten keine. Die mittlere Beobachtungsdauer betrug 33,6 Monate. Primärer Endpunkt war die Gesamtmortalität, als sekundäre Endpunkte wurde der Unterschied in der Gesamtmortalität sowie die Häufigkeit von Krankenhausaufnahmen wegen Herzinsuffizienz oder exazerbierter COPD definiert.

Die Mortalität lag bei Patienten ohne Betablocker höher ( $p=0,039$ im LogRang-Test). Die Einnahme von Betablockern war in einer univariaten Analyse der einzige Faktor, der signifikant mit der Todesrate korrelierte (Hazard Ratio [HR]: 0,41, 95\%-Konfidenzintervall [KI]: $0,17-0,99, p=0,047)$. Die Rate von Dekompensationen der Herzinsuffizienz bzw. Exazerbationen der COPD war unter Carvedilol höher als unter Bisoprolol ( $\mathrm{p}=$ 0,033 im Log-Rang-Test).

In der multivariaten Analyse waren nur häufige Exazerbationen in der Vorgeschichte signifikant mit einer erhöhten Rehospitalisierungsrate wegen Herzinsuffizienz oder COPD verbunden (adjusted HR: 3,11, 95\%-KI: 1,47-6,61; p = 0,003).

- Kubota Y, Asai K, Furuse E et al. Impact of $\beta$-blocker selectivity on long-term outcomes in congestive heart failure patients with chronic obstructive pulmonary disease. Int J Chron Obstruct Pulmon Dis. 2015;10:515-23

\section{KOMMENTAR}

Betablocker können bei kardiologischer Indikation trotz COPD verordnet werden. Das war bekannt [Ni Y et al. J Int Med Res. 2012;40:2051-65]. Was macht diese Studie dennoch Interessant? Es sind drei Dinge: 1. Nach wie vor werden Patienten Betablocker wegen der vermeintlichen Kontraindikation COPD vorenthalten, obwohl die Indikation wegen einer kardialen Erkrankung besteht. Dies hat einen ungünstigen Einfluss auf die Mortalität, was auch in dieser retrospektiven Arbeit beobachtet wurde.

\section{Der Unterscheidung zwischen Asthma} bronchiale und COPD kommt schon wegen der Frage der Komedikation erhebliche Bedeutung zu. Nach wie vor sind Betablocker bei Asthma kontraindiziert, und aller Wahrscheinlichkeit nach wird sich daran auch nichts ändern [Worth H. Pneumologie. 2001;55:53-6]. Indes: Entpuppt sich das vermeintliche Asthma als COPD, so hat die indikationsgerechte Therapie mit Betablockern Relevanz.

3. Die Aussagen zum positiven Effekt der Betablocker bei Patienten, die gleichzeitig an COPD leiden, treffen nur auf die kardioselektiven Substanzen zu. Hier sind in erster Linie Bisoprolol, Metoprololsuccinat und Nebivolol zu nennen, da sie in allen wichtigen Indikationen (arterielle Hypertonie, Zustand nach Infarkt und Herzinsuffizienz) eingesetzt werden können. Wichtig ist: Carvedilol gehört nicht dazu. Allzu oft wird dieser Betablocker falsch als kardioselektiv eingestuft. Dies rührt noch daher, dass Carvedilol der erste Betablocker war, der die Zulassung zur Behandlung der systolischen Herzinsuffizienz erhielt [Packer M et al. N Engl J Med. 1996;334:134955]. Um hier eine deutliche Alleinstellung am Markt zu erreichen und einen Klasseneffekt zu vermeiden, stellte der Hersteller als besondere Eigenschaft die Blockade des peripheren Alpha-1-Rezeptors in den Vordergrund der Kommunikation. Bis heute ist die Verknüpfung „,besonderer Rezeptor - CarvediIol" in unseren Köpfen.

Fazit: "Umparken im Kopf" - die Kampagne eines bekannten Autoherstellers trifft auch auf Carvedilol zu. Diese Substanz muss in unseren Köpfen als Betablocker mit besonderen Eigenschaften umgeparkt werden. Sie gehört zur Klasse der nicht kardioselektiven Betablocker. Bei Patienten, die an COPD leiden, sollte deshalb auf einen anderen Wirkstoff umgestellt werden.

Dr. med. J. de Zeeuw 